\title{
A critical study of the APACHE II scoring system using earlier data collection
}

\author{
M. WATERS, P. NIGHTINGALE \& J. D. EDWARDS \\ Department of Accident and Emergency Medicine, University Hospital of South \\ Manchester, Nell Lane, Withington, Manchester, England
}

\section{SUMMARY}

The APACHE II severity of illness scoring system was prospectively studied on 756 patients admitted to a general intensive care unit (ICU) from January 1986 to June 1988. Admission data were used. Individual and group risk of death were calculated for $=$ 3 diagnostic categories commonly seen in the $A \& E$ department and requiring admission $₫$ to an ICU. The APACHE II score on admission tended to underestimate the risk of death following operative and non-operative trauma, and self-poisoning. This may have been related to the use of an analysis not yet validated against values obtained on admission to ICU. Such validation is urgently needed on a UK population if APACHE II scoring is to be of value in the A\&E department.

\section{INTRODUCTION}

The Acute Physiology And Chronic Health Evaluation (APACHE II) scoring system (Knaus $e t$ al., 1985a) is widely used to assess the severity of illness in patients admitted to an intensive care unit (ICU). Few publications have commented on the logistics of data collection and analysis, tending to concentrate more on validation of the score $₹$ against mortality and possible uses of the system. APACHE II scoring is based on the 9 degree of deviation from normal function of the major organ systems. It is said to be $D$ relatively independent of specific disease processes and therapeutic interventions prior to ICU admission. Knaus validated the system and the risk of death (ROD) calculations $N$ o on the worst recorded value for each parameter in the first $24 \mathrm{~h}$ following ICUN admission. However, he intimated that it would be more logical to base a prognostic index on the earliest available values. We have prospectively studied patients admitted

Correspondence: Dr P. Nightingale, Intensive Care Unit, University Hospital of South Manchester, Withington, Manchester M20 8LR, England. 
to a general ICU whose admission data was obtained in the A\&E department, when these precipitated ICU admission, or the first values collected following admission to ICU from the A\&E department.

\section{PATIENTS AND METHODS}

All patients were seen before or on admission by the ICU consultant on-call. Information was recorded on a standard form and checked by one of us (PN) before being entered into a computerized database for later analysis. Patients admitted electively for repeat haemodialysis and plasmapheresis were not scored. The most immediate reason for admission to ICU was called the primary diagnosis and where this was multiple trauma (operative and non-operative admissions) or self-poisoning, the ROD was calculated using the logistic regression equation and diagnostic category weightings derived by Knaus. Group death rates were obtained by summing the individual risks, and dividing by the number in the group. Actual and predicted death rates were analysed and statistical significance assessed by $95 \%$ confidence bands based on the actual death rates. Death rates relate to hospital mortality. Patients with repeated admissions had ROD calculations performed for the first one only.

\section{RESULTS}

The patient's details, admission characteristics and outcome are shown in Table 1 . During the 30 months studied, there were 756 first admissions to the ICU of which 446 $(59 \%)$ were male and $310(41 \%)$ were female. The median age was 51 years.

Table 1 Admission details of patients given an APACHE II score

\begin{tabular}{cccccc}
\hline \multicolumn{2}{c}{ Number of patients } & & & \multicolumn{2}{c}{ Operative admissions } \\
Male & Female & Age range & Median age & Emergency & Elective \\
\hline $446(59 \%)$ & $310(41 \%)$ & $13-90$ & 51 & $105(13.9 \%)$ & $169(26.4 \%)$ \\
\hline
\end{tabular}

Two hundred and seventy-four (36\%) admissions were operative, of which 105 $(13.9 \%)$ had undergone emergency surgery. There were 289 deaths $(38 \cdot 23 \%)$. The highest APACHE score in a survivor was 40.

Table 2 shows that hospital mortality increased with increasing APACHE II scores.

Table 3 shows that in the three chosen diagnostic category groups, some patients were not surviving despite a calculated ROD of less than $0 \cdot 4$. The actual and predicted death rates are shown and compared using $95 \%$ confidence limits. An unmistakable underscoring by APACHE II is demonstrated in these patients. 
18 M. Waters et al.

Table 2 Mortality rate against admission APACHE II score

\begin{tabular}{|c|c|c|c|c|}
\hline APACHE II score & $\mathbf{N}$ & Alive & Dead & Mortality rate \\
\hline $0-5$ & 204 & 198 & 6 & $2.94 \%$ \\
\hline $6-10$ & 117 & 89 & 28 & $23.93 \%$ \\
\hline $11-15$ & 125 & 79 & 46 & $36 \cdot 8 \%$ \\
\hline $16-20$ & 119 & 61 & 58 & $48 \cdot 74 \%$ \\
\hline $21-25$ & 91 & 22 & 69 & $75.82 \%$ \\
\hline $26-30$ & 47 & 9 & 38 & $80.85 \%$ \\
\hline $31-35$ & 34 & 4 & 30 & $88.24 \%$ \\
\hline $36-40$ & 10 & 5 & 5 & $50 \%$ \\
\hline $41-45$ & 7 & 0 & 7 & $100 \%$ \\
\hline$>46$ & 2 & 0 & 2 & $100 \%$ \\
\hline
\end{tabular}

Table 3 Number of admissions and actual death rates in the three illustrative groups compared with the $\rightarrow$ APACHE II group death rates

\begin{tabular}{|c|c|c|c|c|c|c|}
\hline $\begin{array}{l}\text { Diagnostic } \\
\text { category }\end{array}$ & $\begin{array}{c}\text { Total } \\
\text { number }\end{array}$ & $\begin{array}{l}\text { Number } \\
\text { alive }\end{array}$ & $\begin{array}{c}\text { Number } \\
\text { dead }\end{array}$ & $\begin{array}{l}\text { Actual death } \\
\text { rate }(\%)\end{array}$ & $\begin{array}{c}\text { Group death } \\
\text { rate }(\%)\end{array}$ & $\begin{array}{l}\text { Confidence } \\
\text { limits (\%) }\end{array}$ \\
\hline Overdose & 63 & 52 & 11 & $17 \cdot 5$ & $2 \cdot 2$ & $9 \cdot 05-29 \cdot 1 \gtreqless$ \\
\hline Trauma (op) & 18 & 11 & 7 & 38.9 & $4 \cdot 8$ & $17 \cdot 3-64.25 \overline{6}$ \\
\hline $\begin{array}{l}\text { Trauma } \\
\text { (non-op) }\end{array}$ & 29 & 22 & 7 & $24 \cdot 1$ & $8 \cdot 2$ & $10.30-43.54$ \\
\hline
\end{tabular}

Table 4 Numbers of patients in the largest of the diagnostic category groups

\begin{tabular}{|c|c|c|}
\hline Diagnostic category & Operative admission & Non-operative admission \\
\hline Sepsis & & 78 \\
\hline Post CPR & & 60 \\
\hline Cardiogenic shock & & 23 \\
\hline Asthma & & 22 \\
\hline Aspiration & & 16 \\
\hline Respiratory infection & & 38 \\
\hline Post op. respiratory failure & 20 & \\
\hline GIT neoplasm surgery & 22 & \\
\hline Head trauma & 4 & 13 \\
\hline GIT bleed & 9 & 17 \\
\hline Other cardiovascular & 45 & 9 \\
\hline Other respiratory & 108 & 16 \\
\hline Endocrine/ARF/CRF & 3 & 32 \\
\hline
\end{tabular}




\section{DISCUSSION}

The University Hospital of South Manchester has a general ICU which is a tertiary referral centre for facio-maxillary and plastic surgery, burns and renal medicine.

A profound critique of the logistics of using APACHE is beyond the scope of this paper, but of those relating to the $A \& E$ department, a few deserve mention. It is said that APACHE II scores are relatively free from the effects of therapeutic intervention. During emergency resuscitation, patients are often paralysed, intubated and commenced on mechanical ventilation and anaesthetic drugs will often have to be continued in order for the patient to tolerate endotracheal intubation. Under these circumstances the Glasgow Coma Score and respiratory rate assessments become uninformative when scored at the time of ICU admission. It is not permissible to assess the GCS after these manoeuvres so no neurological points are awarded. Clearly, if the values at the time of arrival in the A\&E department are ignored this will not reflect the true severity of illness in these cases, especially as the GCS is heavily weighted in the Acute Physiology Score (APS). In contrast, the APACHE II system does allow patients who have taken an overdose of sedative drugs to score points on neurological assessment.

When a severely shocked patient is successfully resuscitated in the A\&E department the effect is to reduce the APS, and hence the calculated ROD when this is scored on admission to ICU. Severe derangements of physiology that were evident in the A\&E department may however, even if fully corrected prior to ICU admission, influence eventual outcome. Outcome has been shown to be related to the number and duration of organ system failures, so prompt correction of abnormal physiology should improve outcome (Knaus et al., 1985b). The APACHE II system does not take account of the duration of system failure prior to resuscitation. We strongly suspect that early improvement after initial resuscitation may mask cellular damage sustained by prolonged lowering of the mixed venous oxygen saturation prior to ICU referral, e.g. trauma patients who have been trapped and have a prolonged transit time to hospital, so that a low APS on admission belies the true severity of illness. This is a major disadvantage of single point in time scoring, which can be offset to some extent by both repeated scores and allowing for the rate of change in score from day to day (Chang $e t$ al., 1988).

Paracetamol overdose was one of the commonest reasons for the transfer of selfpoisoned patients to this ICU. Often they had a low APS on arrival in the A\&E department or on admission to ICU, but a significant mortality from hepatic necrosis. They were a markedly different population to the barbiturate, benzodiazepine and opiate overdoses commonly seen in the US and on whom the ROD calculations were validated. The good outcome generally from these overdoses is reflected in the diagnostic category coefficient given to self-poisoning. This may lead to inaccurate ROD calculations with certain types of poisonings and the coefficients may not be applicable in the UK. We feel that coefficients should be derived for different classes of drugs. In using APACHE II we found that there were an inadequate number of diagnostic categories, and would suggest that further developments of the system should address this problem.

We have found the system to underestimate mortality in the groups presented. This could be explained by our use of coefficients based on worst in $24 \mathrm{~h}$ values or differences 
in patient population and referral criteria between the USA and UK. The results of the Intensive Care Society UK APACHE Study should ascertain which, if either, of these two factors are of importance. Knaus has already established that $86 \%$ of worst values were the admission values and it seems to us that some form of APACHE scoring could be useful in the A \& $\mathrm{E}$ department but that USA coefficients are probably inappropriat in a UK population.

\section{REFERENCES}

Chang R., Jacobs S. \& Lee B. (1988) Predicting outcome among Intensive Care Unit patients using computerized trend analysis of daily APACHE II scores. Intensive Care Medicine 14, 558-66.

Knaus W., Draper E., Wagner D. \& Zimmerman J. (1985) APACHE II: A severity of disease classification? system. Critical Care Medicine 13, 818-29.

Knaus W., Draper E., Wagner D. \& Zimmerman J. (1985) Prognosis in acute organ system failure. Annals of Surgery 202, 685-93. 